\title{
Research Data Publishing at UiT The Arctic University of Norway
}

\author{
An Early-Stage Working Paper
}

\author{
Author: Philipp Conzett \\ Affiliation: UiT The Arctic University of Norway \\ ORCID: https://orcid.org/0000-0002-6754-7911
}

Last Updated: $2020-05-17$

\section{Abstract}

This is an early-stage working paper where I share ideas, thoughts, and results from an ongoing project about research data publishing at UiT The Arctic University of Norway. In this initial draft, I'm sharing the results from a small study of how common data publishing is among researchers affiliated with UiT The Arctic University of Norway.

\section{Project information}

Over the last years, there has been made considerable efforts to increase the reproducibility of research results and the reusability of research data. One of the most influential papers in the field of data management saw the light of day in 2016, when a diverse group of stakeholders representing academia, industry, funding agencies, and scholarly publishers postulated a set of principles on how to improve infrastructure supporting the reuse of research data. They referred to these principles as the FAIR Data Principles (Wilkinson et al., 2016). FAIR data are Findable, Accessible, Interoperable, and Reusable.

The project "Research Data Publishing at UiT The Arctic University of Norway" is about how recommendations on how to increase the FAIRness of research data are being adopted in scientific/scholarly communities.

\section{The transition owards increased FAIRness}

As a first step, I wanted to know how common data publishing is among researchers. The idea for this was inspired by a presentation by Brian Nosek on how the transition towards increased FAIRness requires change in research culture (Nosek, n.d.). Nosek uses two theories to illustrate the mechanisms and progress involved in research culture change. The first one is the Theory of Diffusion of Innovation, proposed by Everett Rogers in 1962 (Rogers, 2003). According to this view, a change or innovation in research culture is first initiated and spearheaded by a small group of innovators, followed by a somewhat larger group of early adopters, before the change is adopted by an early and afterwards by a late majority of members of the research community. Finally, the change also spreads to the last group of adopters, called laggards in Rogers' theory. 
The second theory adopted by Nosek is the Theory of Human Motivation, proposed in 1943 by Abraham Maslow (Maslow, 1943). Maslow postulates a hierarchy of needs to illustrate how human behaviour is governed by motivation. Adopting Maslow's hierarchy of needs to the realm of research, Nosek uses a pyramid to illustrate the different motivational factors and driving forces behind cultural change. In this view, basic infrastructure including tools and skills are necessary to make change in research culture possible. Turning this infrastructure more userfriendly, makes it easy for members of the research community to adopt new practice. Once new practice has spread to and is recommended by (a large part of) a research community, its adoption may be considered normative. As a further step in advancing the uptake of new practice, incentives may be introduced that make the adoption of the practice rewarding. Finally, the implementation of new practice may be made required by policies.

In Figure 1, Nosek combines his adaption of Rogers' Theory of Diffusion of Innovation and Maslow's hierarchy of need. In this view, basic infrastructure making the change of research culture possible is sufficient motivation for the small group of innovators to adopt new practice. Early adopters go along with the innovators once adoption has been made easy. Adoption by the early majority is mainly driven by the new practice becoming part of community norm, while rewarding incentives are the main motivation needed for the late majority to change their behaviour. Finally, policy requirements seem to be the last resort to motivate the group of laggards to comply with what by then probably is recognized as a de facto standard in the research community.

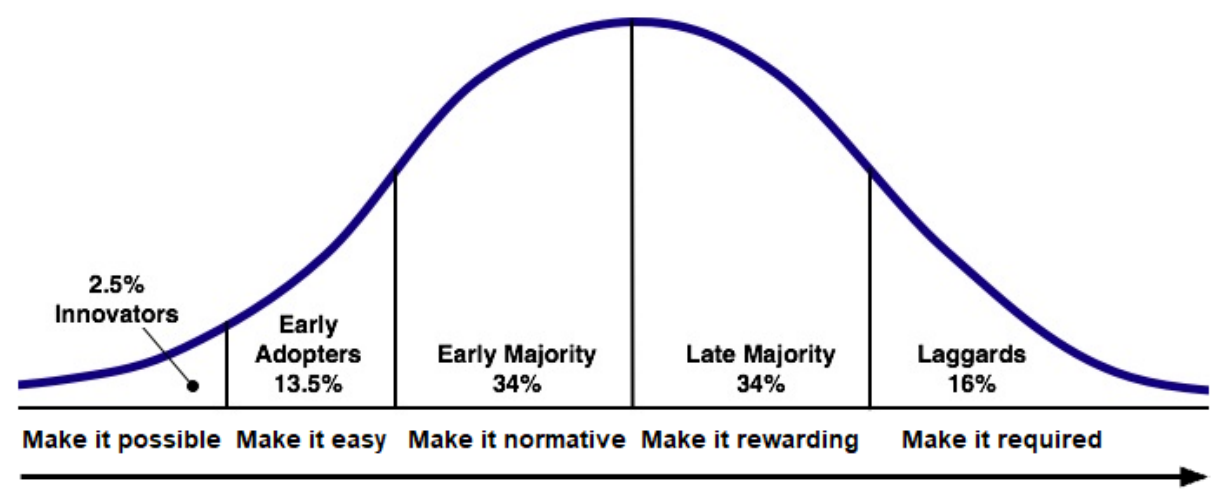

Figure 1: Change in research culture: diffusion and motivation. Slightly adapted from Nosek (n.d.), licensed under $\underline{\text { CCO }}$ 1.0 Universal.

To my knowledge, Nosek's view of how change in research culture diffuses in research communities has not been verified by empirical data. However, applied to the process of increasing the FAIRness of research data, there are some observations and a small empirical study I have made in my work with providing support services for research data management for the last six years which to some degree substantiate Nosek's view.

First, there are parts of research communities that started adopting the FAIR Data Principles without there being other resources in place than the basic infrastructure making the adoption possible. Parts within the bioinformatics community may here serve as an example.

Second, for quite some time researchers have had access to data repositories and other support services making it rather easy to make their data at least partly FAIR. Nosek suggests that - seen from a post-hoc perspective once the culture change has been accomplished - the group of early 
adopters amounts to $13.5 \%$ of the research community. To get a rough indication of how large the group of early adopters of the FAIR Data Principles currently might be in Norway, one could compare the number of unique authors of published datasets with the number of unique authors of publications of research results in anthology chapters, articles and monographies (books). In a small case study, I obtained these numbers limited to researchers affiliated with my own university, UiT The Arctic University of Norway (UiT), and limited to outputs published in 2019. The background data for this small investigation including a description of the methods and tools used to obtain these numbers are available in Conzett (2020). There were 20 unique UiT-affiliated researchers who published one or more datasets in 2019. In the same year, there were 1736 unique UiT-affiliated researchers who published research results in recognized publishing venues. The group of UiT-affiliated researchers who published data in 2019 may thus be said to represent $1.15 \%$ of all UiT-affiliated researchers who published research results in the same year. These numbers are based on a very small selection of researchers and limited to the span of only one year. I still argue that they can give us a rough indication. The results from this small investigation suggest that the percentage of researchers making use of easily accessible resources to make data more FAIR is still much lower than the $13.5 \%$ indicated by Nosek for the group of early adopters.

Third, there seems to be considerable agreement among research data professionals that strong community support and recommendations as well as rewarding incentives are crucial to advance the further uptake of the FAIR Data Principles. On the other hand, the role of policy requirements is maybe not as straightforward. Some research funders and journals have introduced policies that require researchers to make their data openly available. Such policies are most effective when non-compliance results in direct negative consequences like repayment of funding or the preclusion of article or book manuscripts from being accepted for review. Journals' data policies are thus an effective incentive for researchers to make their data available. Currently, most universities do not reinforce the compliance with their research data policies. However, institutional policies are still important as they help provide useful legitimation for institutional support services.

[Further steps to come.]

\section{References}

Conzett, P. (2020). Research Data Publishing at UiT The Arctic University of Norway (Version 1) [Dataset]. DataverseNO. https://doi.org/10.18710/JWTJJB

Maslow, A. H. (1943). A theory of human motivation. Psychological Review, 50(4), 370-396. https://doi.org/10.1037/h0054346

Nosek, B. (n.d.). Shifting Incentives from Getting It Published to Getting it Right. Retrieved 4 April 2020, from https://osf.io/bxjta/

Rogers, E. M. (2003). Diffusion of innovations (5th ed., pp. XXI, 551). Free Press.

Wilkinson, M. D., Dumontier, M., Aalbersberg, Ij. J., Appleton, G., Axton, M., Baak, A., Blomberg, N., Boiten, J.-W., Santos, L. B. da S., Bourne, P. E., Bouwman, J., Brookes, A. J., Clark, T., Crosas, M., Dillo, I., Dumon, O., Edmunds, S., Evelo, C. T., Finkers, R., ... Mons, B. (2016). The FAIR Guiding Principles for scientific data management and stewardship. Scientific Data. https://doi.org/10/bdd4 